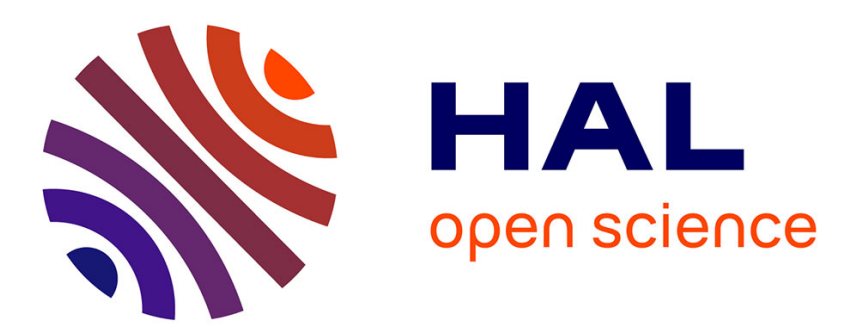

\title{
Optical Incoherence Tomography: a method to generate tomographic retinal cross-sections with non-interferometric adaptive optics ophthalmoscopes
} Pedro Mecê, Elena Gofas-Salas, Michel Paques, Kate Grieve, Serge Meimon

\section{- To cite this version:}

Pedro Mecê, Elena Gofas-Salas, Michel Paques, Kate Grieve, Serge Meimon. Optical Incoherence Tomography: a method to generate tomographic retinal cross-sections with non-interferometric adaptive optics ophthalmoscopes. Biomedical optics express, 2020, 11 (8), pp.4069. 10.1364/BOE.396937 . hal-02895839

\section{HAL Id: hal-02895839 \\ https://hal.science/hal-02895839}

Submitted on 10 Jul 2020

HAL is a multi-disciplinary open access archive for the deposit and dissemination of scientific research documents, whether they are published or not. The documents may come from teaching and research institutions in France or abroad, or from public or private research centers.
L'archive ouverte pluridisciplinaire HAL, est destinée au dépôt et à la diffusion de documents scientifiques de niveau recherche, publiés ou non, émanant des établissements d'enseignement et de recherche français ou étrangers, des laboratoires publics ou privés. 


\title{
Optical Incoherence Tomography: a method to generate tomographic retinal cross-sections with non-interferometric adaptive optics ophthalmoscopes
}

\author{
Pedro Mecê, ${ }^{1,},{ }^{*}$ (D) Elena Gofas-Salas, ${ }^{2}$ (1) Michel Paques, $, 2,3$ \\ Kate Grieve, ${ }^{2,3}$ and Serge Meimon ${ }^{4}$ \\ ${ }^{1}$ Institut Langevin, ESPCI Paris, CNRS, PSL University, 1 rue Jussieu, 75005 Paris, France \\ ${ }^{2}$ Quinze-Vingts National Eye Hospital, 28 Rue de Charenton, Paris, 75012, France \\ ${ }^{3}$ Institut de la Vision, Sorbonne Université, INSERM, CNRS, F-75012, Paris, France \\ ${ }^{4}$ DOTA, ONERA, Université Paris Saclay F-91123 Palaiseau, France \\ *pedro.mece@espci.fr
}

\begin{abstract}
We present Optical Incoherence Tomography (OIT): a completely digital method to generate tomographic retinal cross-sections from en-face through-focus image stacks acquired by non-interferometric imaging systems, such as en-face adaptive optics (AO)-ophthalmoscopes. We demonstrate that OIT can be applied to different imaging modalities using back-scattered light, including systems without inherent optical sectioning and, for the first time, multiply-scattered light, revealing a distinctive cross-sectional view of the retina. The axial dimension of OIT cross-sections is given in terms of focus position rather than optical path, as in OCT. We explore this property to guide focus position in cases where the user is "blind" focusing, allowing precise plane selection for en-face imaging of retinal pigment epithelium, the vascular plexuses and translucent retinal neurons, such as photoreceptor inner segments and retinal ganglion cells, using respectively autofluorescence, motion contrast and split detection techniques.
\end{abstract}

(C) 2020 Optical Society of America under the terms of the OSA Open Access Publishing Agreement

\section{Introduction}

High-resolution in-vivo imaging of the human retina can be achieved using Adaptive Optics (AO) ophthalmoscopes, such as Flood-Illumination Ophthalmoscopes (FIO) [1] and Scanning Laser Ophthalmoscopes (SLO) [2], owing to the capacity of AO to measure and correct for static and dynamic monochromatic ocular aberrations in real-time [3,4]. Such high-resolution retinal images play an important role in early-stage retinal disease diagnosis, monitoring the progression of retinal disease and the effect of new therapeutic drugs. [2,5].

To be able to explore the retinal volume using $\mathrm{AO}$ ophthalmoscopes, the control of the imaging focal position becomes crucial. At present, the positioning of the image focal plane is generally done empirically by visualizing the en-face images displayed in real-time and judging sharpness of the retinal image. This focus guidance approach seems sufficient when using confocal AO-SLO to image hyper-reflective retinal layers such as photoreceptors and nerve fiber layer (NFL) [2], especially due to the optical sectioning capability of such imaging systems. Nevertheless, the same cannot be said when using nonconfocal imaging modalities as AO-FIO and AO-SLO split-detection [6], multi-offset [7], motion contrast [8], and autofluorescence [9], since displayed images present a weak signal-to-noise ratio (SNR) and a low contrast, and users are mostly "blind" focusing. As a result, the acquisition of image stacks around the retinal layer of interest, i.e. for different focal planes, and the assessment of image quality after acquisition, to select the best image stack, are mandatory, time-consuming steps which are not always compatible with the clinical environment. To avoid these drawbacks, a focus-guidance tool becomes essential, especially to reveal hypo-reflective or transparent structures such as cone photoreceptor inner 
segments (IS) [6], retinal ganglion cells (RGC) [7], perfusion in microvasculature [8,10] or those masked by neighboring structures of high reflectivity such as retinal pigment epithelium (RPE) lying beneath photoreceptors [9].

To better manage focus position, some early work involved acquisition of through focus image stacks using SLO to understand topography and to select an image at a particular layer [11,12]. Inspired by autofocus systems [13], focus measure operators were used to characterize image quality while spatial filtering was proposed to improve image quality when imaging photoreceptors using AO-FIO [14,15]. However, previous methods using AO-FIO were only able to guide focus position when imaging hyper-reflective retinal structures, such as photoreceptors.

Here, we present Optical Incoherence Tomography (OIT): a digital method to generate tomographic retinal cross-sections from through-focus en-face image stacks (otherwise known as depth stacks, axial stacks or Z-stacks) acquired by non-interferometric AO-ophthalmoscopes. Contrary to Optical Coherence Tomography (OCT) [16], which is sensitive to the optical path difference between the reference and the sample arms, OIT produces cross-sections as a function of the focal position, making it highly valuable as a focus guidance tool. We apply OIT to different AO-ophthalmoscope modalities such as AO-FIO, confocal AO-SLO, split-detection AO-SLO and motion contrast AO-SLO. We demonstrate that although cross-sectional image quality is inferior, most of the retinal layers commonly resolved by OCT cross-sections [16] can nevertheless also be resolved and identified in OIT cross-sections. We use the focus sensitivity of the OIT method to precisely guide focus positioning, facilitating imaging of the RPE, all retinal vascular plexuses, and transparent neurons such as photoreceptor IS and RGC when using respectively autofluorescence, motion contrast, and split detection techniques.

\section{Methods}

\subsection{The Optical Incoherence Tomography procedure}

The OIT method is composed of three steps: Z-stack acquisition, image filtering and computation of the image energy.

Z-stack acquisition: Acquisition of en-face images from different focal planes, forming a Z-stack (or a fly-through movie) in the $\mathrm{z}$ direction. Here, the Z-stacks were acquired in a step-wise manner, by manually changing the focus position and acquiring images at each plane. An entire Z-stack was acquired in around 20 minutes.

En-face image filtering: After Z-stack acquisition, each en-face image from the Z-stack has its low-spatial frequency content filtered out. Indeed, since nonconfocal imaging systems do not present inherent optical sectioning, i.e. the capacity to reject out-of-focus photons, the high-pass filter enables one to take advantage of the fact that high-spatial frequency is only present for photons coming from the in-focus plane [17], creating an axial sectioning effect. Here, we chose to use an order 2 Butterworth filter to avoid adding high-spatial frequency artifacts to images. The high-pass filter cut-off frequency choice, and its implications, is explained in Sect. 3.1.

En-face image energy computation: To obtain OIT cross-sectional images, similar to an OCT "B-scan", each en-face image from the Z-stack, after spatial filtering, is divided into an overlapping grid of $m \times n$ pixel regions of interest (ROIs), in the lateral dimension (x,y), where each ROI is displaced from the previous by 1 pixel in the $\mathrm{x}$ direction (i.e. in the $\mathrm{m}$ direction). To favor a trade-off between point-wise accuracy and smoothness, we used two ROIs to generate the OIT cross-sections. The first ROI was composed of $4 \times 40$ pixels $(3 \mu \mathrm{m} \times 30 \mu \mathrm{m}$ in the retinal plane), favoring the high lateral resolution brought by the use of $\mathrm{AO}$, thus favoring the contrast of small retinal features. The size of this ROI was chosen based on photoreceptor spacing (in the $\mathrm{x}$ direction) and to increase OIT image contrast. The second ROI was composed of $40 \times 40$ pixels (30 $\mu m \times 30 \mu m$ in the retinal plane), favoring the detection of contrast between en-face images of the Z-stack and the appearance of bands in the OIT cross-section defining the retinal layers. 
The energy of each ROI $\left(E_{R O I}\right)$ is then computed as follows:

$$
E_{R O I}=\sum_{u} \sum_{v}\left|\widetilde{I_{R O I}}(u, v)\right|^{2}
$$

where $\widetilde{I_{R O I}}$ is the Fourier Transform of the ROI from each filtered en-face image from the Z-stack. Owing to the image filtering step, high energy values will be obtained in ROIs presenting a significant amount of high spatial frequency content. On the other hand, out-of-focus structures and in-focus structures presenting a low spatial frequency content will present a low energy value as low spatial frequency was filtered out. Note that filtering and energy computation can in practice be implemented in a single step, though they are separated here for clarity. Each energy measurement is then allocated to a coordinate $(\mathrm{x}, \mathrm{z})$ generating the OIT cross-section (see Visualization 1 for an animation depicting the energy measurement step in detail). For both ROIs, an OIT cross-section is generated, normalized and then averaged to favor, at the same time, the contrast of small retinal features and retinal layers as mentioned above. Finally, we made available an open source Matlab GUI where the user can load a Z-stack and generate its OIT cross-section in a few seconds, and where OIT parameters such as the size of the ROI and the cut-off frequency of the high-pass filter can be modified [18].

\subsection{Imaging acquisition and processing}

The Z-stacks necessary to generate OIT cross-sections were obtained using the PARIS AO-FIO and a modified version of the MAORI (multimodal adaptive optics retinal imager) AO-SLO (Physical Sciences, Inc., Andover, MA, USA). Both systems were described in detail elsewhere [1,9]. The Z-stack from the PARIS AO-FIO was obtained by translating the imaging camera parallel to the optical axis with a constant step of $30 \mu \mathrm{m}$ in the retinal plane. AO-SLO Z-stacks were obtained by adding constant defocus values to the deformable mirror $[9,19]$. The equivalent axial displacement was approximately $20 \mu \mathrm{m}$ for Fig. 2 and $15 \mu \mathrm{m}$ for Figs. 4,5. The aperture of the PARIS AO-FIO and the MAORI AO-SLO were respectively limited to $5 \mathrm{~mm}$ and $7 \mathrm{~mm}$ diameter at the pupil plane, giving a theoretical depth of field at the diffraction-limit of around $50 \mu \mathrm{m}$ and $25 \mu \mathrm{m}$ respectively [20]. The AO-loop rates of the PARIS AO-FIO and MAORI AO-SLO were set to $50 \mathrm{~Hz}$ and $25 \mathrm{~Hz}$ respectively.

Image acquisition was performed on two healthy subjects aged 25 and 38. Research procedures followed the tenets of the Declaration of Helsinki. Informed consent was obtained from subjects after the nature and possible outcomes of the study were explained. The study was authorized by the appropriate ethics review boards (CPP and ANSM (IDRCB numbers: 2016A00704-47 and 2019-A00942-55)). Before the acquisition, pupil dilation and accommodation paralysis were performed by introducing one drop of each Tropicamide and Phenylephrine 10\%, assuring a constant pupil diameter and minimal interference of defocus dynamics due to natural accommodation during Z-stack acquisition [4,21]. Subjects were seated in front of the systems, stabilized with a chin and forehead rest and asked to fixate a focused target. For each focal plane, a total of 100 images were acquired. When using the AO-SLO device, the deformable mirror focal plane position was recorded along with simultaneously captured confocal and split-detection image stacks. In this configuration, the AO-SLO light level entering the eye from the illumination source and the wavefront sensor laser beacon was respectively $0.95 \mathrm{~mW}$ and $0.7 \mathrm{~mW}$. For the PARIS AO-FIO, the total power entering the eye from the illumination source and the wavefront sensor laser beacon were respectively $350 \mu \mathrm{W}$ and $1.8 \mu \mathrm{W}$. For both imaging systems, the light level was below the ocular safety limits established by the ISO standards for group 1 devices.

Following the acquisition, to correct for fixational eye motion [22], non-rigid registration (for the AO-SLO images) [23] and normalized cross-correlation registration (for AO-FIO images) were performed on each image sequence for a given focal plane. After registration, we selected 10 out of the 100 images of best quality, as computed by the image energy [4]. Then the 10 
selected images were averaged or, in the case of split-detection images, had the temporal standard deviation computed to extract perfusion maps [8], providing the final Z-stack used to generate the OIT retinal cross-sections. Before performing the OIT procedure (Sect.2.1, above), images composing the Z-stacks were registered (i.e. spatially aligned) pairwise. Because the Z-stacks used to generate the OIT cross-sectional images were composed of 20 to 30 en-face images, resulting in a cross-section with only 20 to 30 pixels in the axial (z) direction, we resized the OIT cross-sections in the axial (z) direction by using bilinear interpolation and increasing the number of pixels by a factor of 15 , thus facilitating OIT cross-section visualization and analysis. Finally, generated OIT cross-sections were used in two different ways (specified for each case throughout Sect.3): to determine the retinal depth location of an en-face image from the original Z-stack; or to precisely adjust the focus of the AO-ophthalmoscope to acquire a sharp en-face image in a retinal layer of interest, the latter case using the previously acquired OIT cross-section as a focus guidance tool.

\section{Results}

\subsection{OIT axial sectioning capacity}

A crucial step of the OIT procedure consists of filtering out the low-spatial frequency content. Indeed, since nonconfocal imaging systems do not present inherent optical sectioning, i.e. the capacity to reject out-of-focus photons, the high-pass filter enables one to take advantage of the fact that high-spatial frequency is only present for photons coming from the in-focus plane [17], creating an axial sectioning effect. To demonstrate the axial sectioning capability of the OIT and the impact of the choice of cut-off frequency, we applied the OIT procedure to a Z-stack obtained from a USAF target using the PARIS AO-FIO. Figures 1(a-d) present the area of one en-face image where the OIT cross-section was generated, and OIT cross-sections obtained without spatial filtering and with spatial filtering for a $10 \%$ and $50 \%$ normalized cut-off frequency.

The choice of cut-off frequency will induce two different behaviors. On the one hand, by increasing the cut-off frequency, the axial sectioning ability of OIT is enhanced. Figure 1(d) outlines this behavior by presenting the axial sectioning, computed through the full-width at half maximum (FWHM) in the axial direction of OIT cross-sections, as a function of the normalized cut-off frequency. Note that the sectioning ability is limited by the depth of field (DOF) at the diffraction limit, which can be defined as $\frac{2 \lambda}{N A^{2}}$ (where $\lambda$ is the illumination source wavelength and $N A$ the numerical aperture) [20]. The DOF defines the depth over which the best focus can be achieved. Outside of this depth, the high spatial frequency content starts to lose contrast, and, consequently, image sharpness reduces [20]. On the other hand, as the cut-off frequency is increased, the OIT cross-section loses contrast and SNR (Fig. 1(e)). This latter effect happens since the contrast is gradually reduced towards zero at a point defined by the lateral resolution of the optical imaging system [20]. We can expect to obtain sufficient OIT image contrast (higher than $80 \%$ ) for normalized cut-off frequencies ranging from $1 \%$ to $25 \%$ of the diffraction limited cut-off frequency. To distinguish different retinal layers, taking into account their spatial frequency content, we decided to use a high-pass filter with a normalized cut-off frequency of $10 \%$ of the diffraction limited cut-off frequency, representing a good trade-off between the sectioning ability $(1.3 \times \mathrm{DOF})$ and the image contrast. For example, with this cut-off frequency, for a 7-mm diameter pupil and a light source of $850 \mathrm{~nm}$, we expect to achieve an axial sectioning of $32.5 \mu \mathrm{m}$ at the diffraction limit.

While OCT cross-sections' axial dimension (z) is given by the optical path difference between the sample and reference arms, OIT cross-sections' axial dimension (z) is graduated in defocus. Therefore, OIT can be used as a tool to measure and control the focus position. Here, in the case of the USAF target, one can precisely determine beforehand the position of the imaging plane to extract a sharp image of the USAF target. 

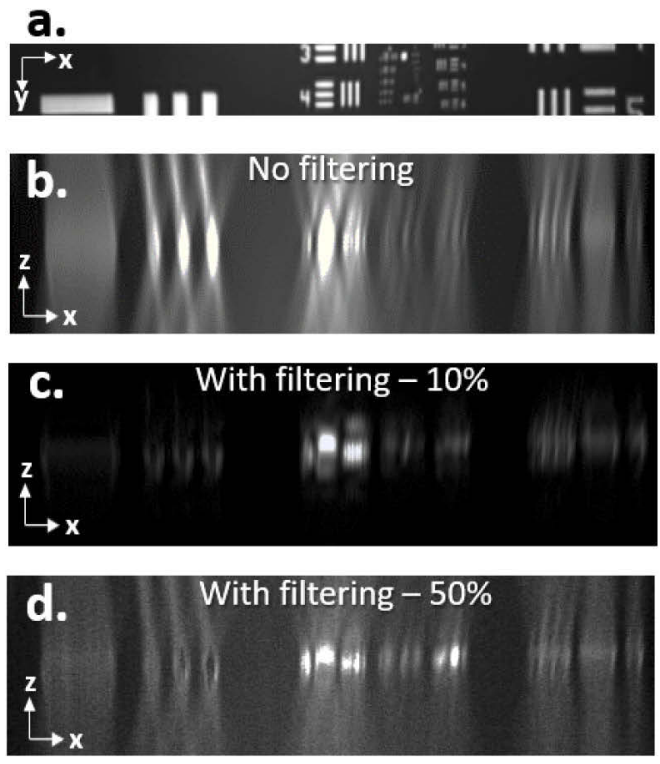

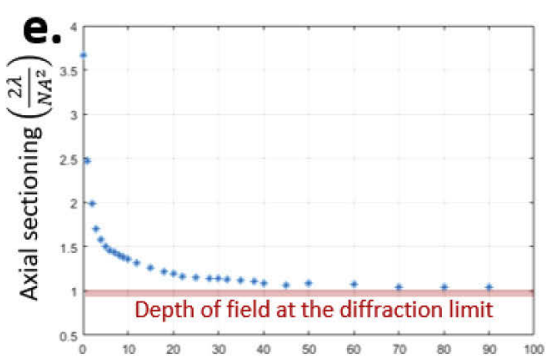

Normalized cut-off frequency (\%)

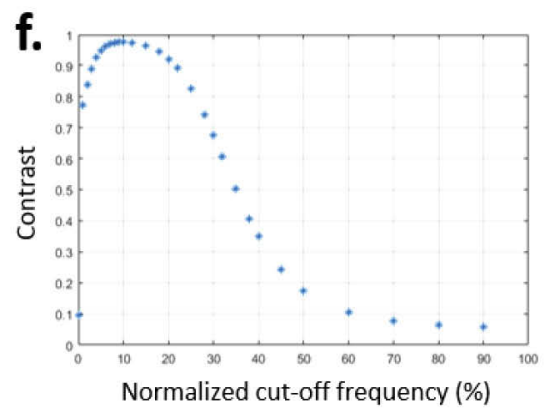

Fig. 1. a. Area of the en-face image where the OIT method was applied. b. Generated OIT cross-sections without spatial filtering. c,d. Generated OIT cross-section after applying a high-pass spatial filtering with a normalized cut-off frequency of $10 \%$ and $50 \%$ respectively. Note that, although at $50 \%$ a better axial sectioning is achieved, the OIT cross-section presents a better contrast for $10 \%$ cut-off frequency. e. The axial sectioning capacity, given in terms of depth of field $\left(\frac{2 \lambda}{N A^{2}}\right)$ at the diffraction limit, as a function of the normalized cut-off frequency. f. The contrast of OIT cross-section as a function of the normalized cut-off frequency.

\subsection{OIT applied to AO ophthalmoscopes}

Figure 2(a,c) presents OIT tomographic retinal cross-sections acquired at $7^{\circ}$ Nasal using the confocal AO-FIO and AO-SLO. We compare both OIT cross-sections with an OCT image acquired at the same retinal location with Spectralis OCT (Heidelberg Engineering, Germany, Fig. 2(b)). Although OCT can achieve a far better axial resolution than OIT (i.e. not limited by DOF but by the light source bandwidth), most of the retinal layers commonly resolved in OCT can nevertheless be identified in both AO-SLO and AO-FIO OIT cross-sections, namely: 1) the NFL (blue arrow), which gets thicker as eccentricity increases in the OCT image (Fig. 2(b)); 2) two intermediate layers labeled, so far, as (1) and (2) (see Sect. 4.4); 3) inner/outer segment junction (IS/OS, green arrow) and cone outer segment tips (COST) (orange arrow). Note that layer appellations are assigned based on comparison with OCT, and as such follow OCT nomenclature.

The proposed retinal layer labeling can be further confirmed by looking at the stack of en-face images, used to generate the OIT cross-section (Figs. 2(d,e)). Note that at the focus positions corresponding to NFL and IS/OS layers, sharp images of these two retinal structures were acquired. En-face images acquired at the focus positions corresponding to intermediate layers (1) and (2) present images with defocused (not sharp) NFL and IS/OS respectively. The origin of these two bands is discussed in Sect. 4.4. The area of the en-face image where the OIT tomographic retinal cross-sections were generated, comprising a retinal vessel, is indicated by the white dashed-rectangle in NFL en-face images in Figs. 2(d,e). It is possible to identify the vessel present within the white dashed-rectangle on the OIT and OCT cross-sections (red arrows) and conclude on its axial position, here at the superficial vascular plexus (SVP). Owing to the high 

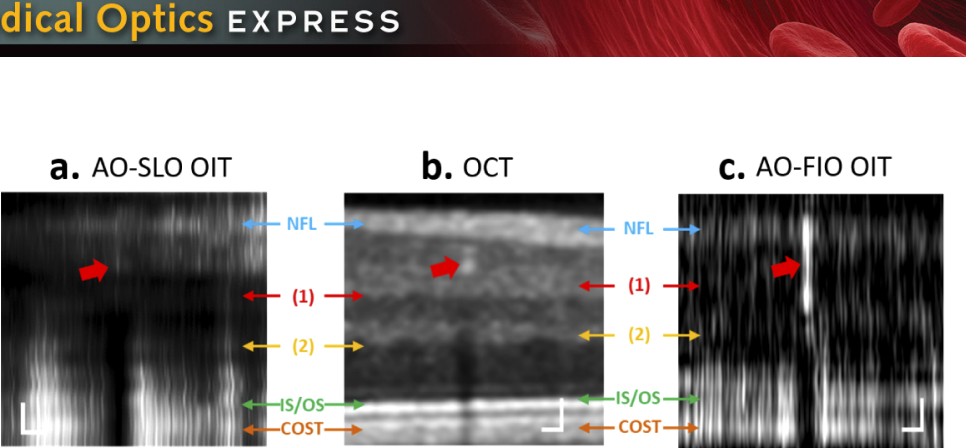

d.
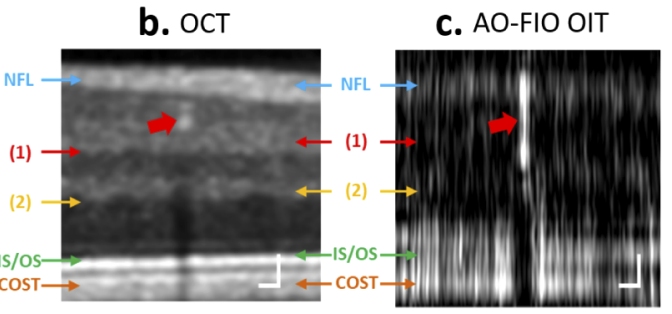

AO-SLO images

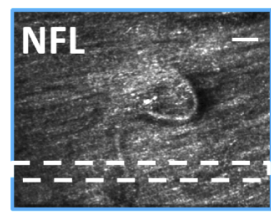

e.
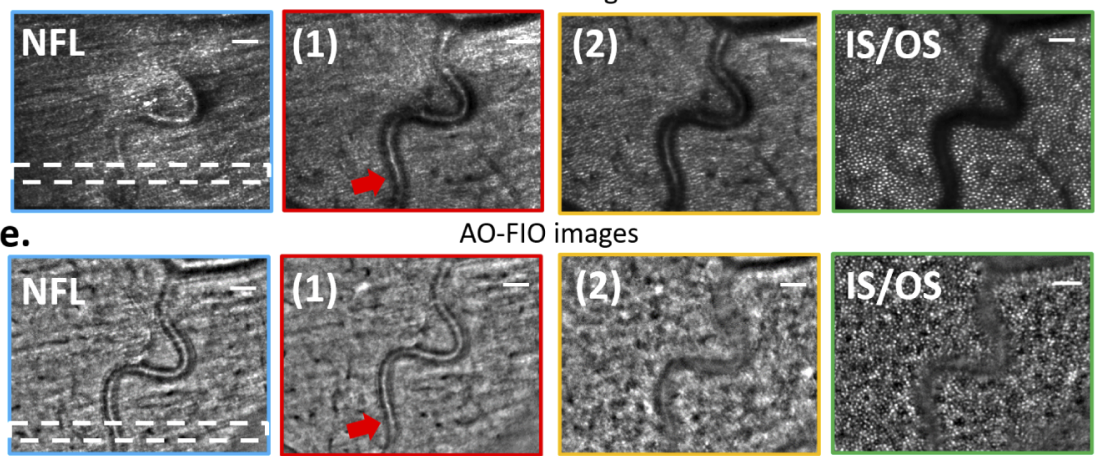

Fig. 2. a-c Tomographic retinal cross-sections generated by, respectively, AO-SLO OIT, OCT, and AO-FIO OIT for the same subject and retinal location, where the main retinal layers can be identified. d,e En-face retinal images, from the original Z-stack, obtained when precisely positioning the focal plane at the layers labelled NFL, (1), (2) and IS/OS, guided by OIT cross-sectional images. White-dashed rectangle: Area of the en-face image where OIT cross-sections were extracted. Red arrows: vessel location. AO-FIO en-face images had their background subtracted [1]. (a,b,c) Log scale. Scale bar: $50 \mu m$.

lateral resolution of $\mathrm{AO}$ ophthalmoscopes, interconnecting capillaries [24,25], linking different capillary plexus, can be visualized in OIT cross-sections as highlighted in Visualization 2.

\subsection{RPE autofluorescence imaging guided by OIT}

We used the AO-SLO OIT to precisely adjust the focus position of the system to the COST layer and new images were acquired using confocal AO-SLO and autoflourescence AO-SLO. While confocal AO-SLO en-face images present photoreceptors (Fig. 3(a)), the detection of the autofluorescence signal in AO-SLO reveals the RPE [9] (Fig. 3(b)). Indeed, since the axial resolution of the AO-SLO is not sufficient to separate COST and RPE, a sharp image of the RPE autofluorescence is produced at the COST focal position. Zoomed areas and the power spectral density (PSD) of Figs. 3(a,b) are also shown. The radial averaged PSD for both confocal and autofluorescence AO-SLO images acquired simultaneously at the RPE focal position (Fig. 3(c)) gives characteristic peaks of cone density of $18000 \mathrm{cells} / \mathrm{mm}^{2}$ and RPE density of 5000 cells $/ \mathrm{mm}^{2}$, consistent with previous studies for $7^{\circ}$ eccentricity $[9,26]$. The axial distance between the IS/OS junction and the COST for OIT cross-sections of both AO-SLO and AO-FIO was approximately $30 \mu \mathrm{m}$, in accordance with in-vivo OCT and AO-OCT data [27].

\subsection{D microvasculature imaging guided by OIT}

Figures $4(\mathrm{a}, \mathrm{b})$ present a comparison between OIT cross-sections generated through confocal AO-SLO and nonconfocal split detection AO-SLO at $7^{\circ}$ Nasal. Colored arrows in OIT crosssections indicate the focus position where en-face images from the original Z-stack, presented in 
a. Confocal AO-SLO
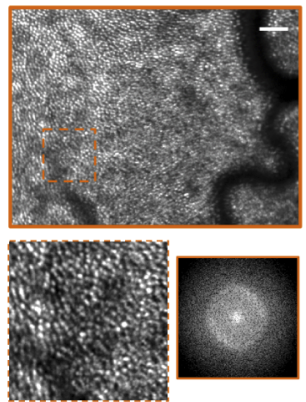

b. Autofluorescence AO-SLO

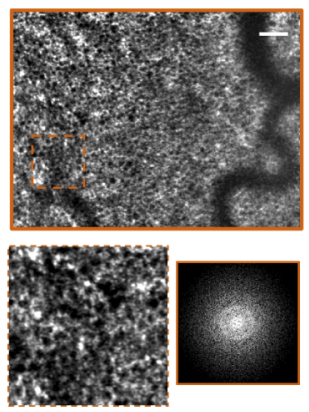

C.

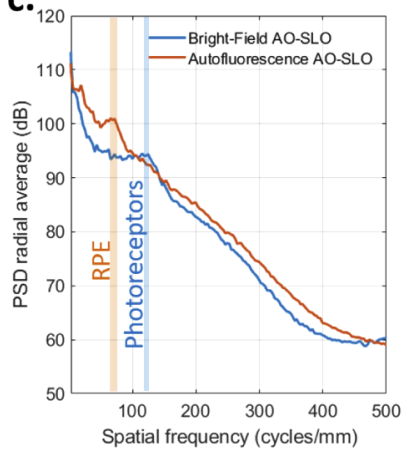

Fig. 3. a,b En-face retinal images obtained using respectively confocal and autofluorescence AO-SLO. The AO-SLO focal plane was adjusted to the COST focal position prior to acquisition using the previously generated OIT cross-section (Fig. 2(a)). The RPE signal is masked in the confocal channel by highly reflective photoreceptor signal due to poor axial resolution, hence the need for autofluorescence. PSD of en-face zoomed images (orange dashed-square) are also given. c. PSD radial average of previously presented zoomed areas outlining the spatial frequency of the photoreceptor and RPE mosaics respectively. Scale bar: $50 \mu m$.

Fig. 4(d,e), were acquired. White dashed rectangles indicate the area of the en-face image where OIT cross-sections were generated.

Two main differences can be noticed when producing split detection OIT cross-sections compared to those generated from confocal images. The first difference concerns the NFL layer which seems to disappear in split detection OIT, indicating, as previously stated, that NFL becomes mostly transparent in multiply scattered light modalities [8]. Secondly, retinal layers in the inner retina get brighter with split detection OIT. By producing the OIT corresponding to a Z-stack of perfusion map images (Fig. 4(c)), we can deduce that these layers correspond to vascular plexuses, of which we expect there to be four at $7^{\circ}$ Nasal [24,28]. Using the split-detection OIT to precisely position the focal plane, we were able to extract perfusion maps of each of the four vascular plexuses (Fig. 4(f)), named according to [24]: radial peripapillary capillary plexus (RPCP), superficial vascular plexus (SVP), intermediate vascular plexus (IVP) and deep vascular plexus (DVP). Owing to the precise location of focus position, one can generate depth-color coded perfusion maps with ease, revealing the 3D organization of the vascular network (Fig. 4(g)). Figure 5 presents the same comparison and results but at $7^{\circ}$ Temporal, where NFL is less dense and all three expected vascular plexuses [28] are visible in the split-detection OIT.

Table 1 gives the axial distance between consecutive plexuses measured through OIT crosssections of Figs. 4(b) and 5(b). All measured values, and the fact that the IVP is closer to DVP in Temporal position compared to Nasal, are consistent with in-vivo OCT angiography data in previous studies [24,28,29]. Full Z-stacks used to generate the OIT images of Figs. 4,5 can be visualized in Visualization 3 and Visualization 4.

\subsection{Using OIT to guide imaging of transparent retinal neurons}

Another interesting difference between OIT cross-sections generated by confocal and splitdetection modalities is the presence of a retinal layer, just above the IS/OS, that gets brighter with split detection OIT (Figs. 4(a,b), 5(a,b)). By using OIT to position the focal plane at this layer in the original Z-stack, we were able to precisely image photoreceptor IS in both imaging locations (Fig. 6). On the one hand, for the split detection modality, note that the photoreceptor 
a. Confocal OIT

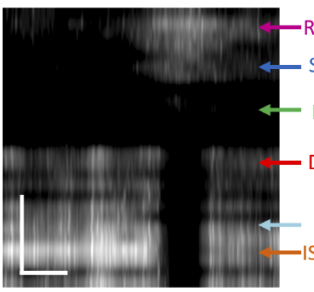

d.

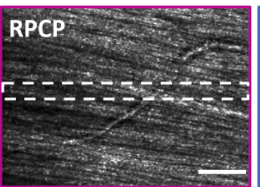

e.

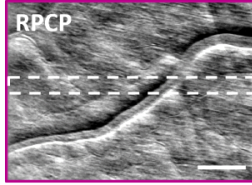

f.
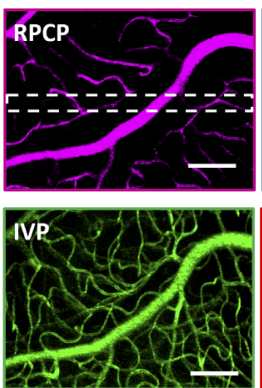

b. Split Detection OIT

C. Perfusion Map OIT

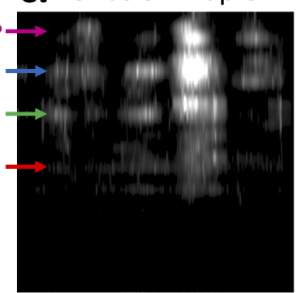

Confocal images
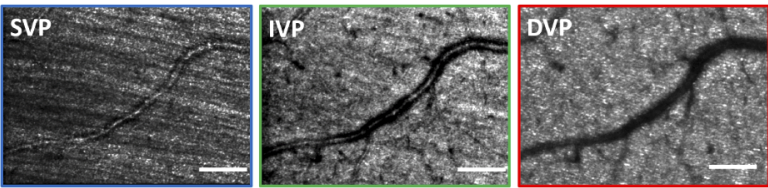

Split Detection images
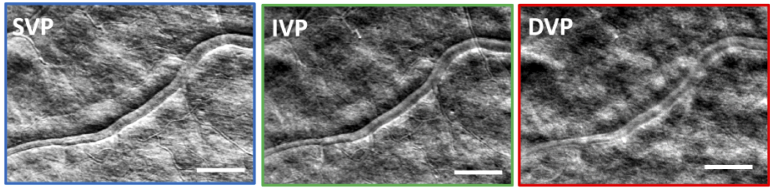

g.
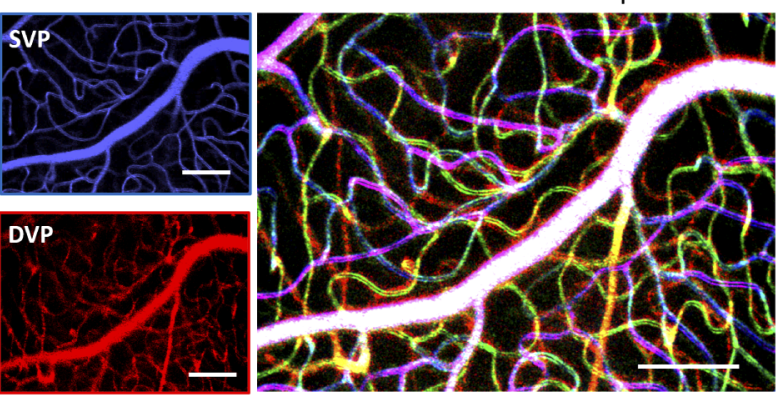

Fig. 4. a-c Tomographic retinal cross-sections generated by, respectively, confocal, split detection, and motion contrast techniques in AO-SLO for the same subject at $7^{\circ}$ Nasal where the NFL is dense and four vascular plexuses can be seen. d-f En-face retinal images from the original Z-stack obtained when precisely positioning the focal plane at the layers labeled RPCP, SVP, IVP and DVP, with the help of OIT method, using respectively confocal, split-detection and motion contrast techniques. $\mathrm{g}$ Composite perfusion map image, revealing the 3D organization of the retinal vascular network. White-dashed rectangle: Area of the en-face image where OIT cross-sections were extracted. (a) Log scale. (b,c) Linear scale. Scale bar: $100 \mu m$.

Table 1. Axial distance between vascular plexuses measured through OIT cross-sections. Four and three plexuses are found at $7^{\circ}$ nasal and $7^{\circ}$ temporal respectively.

\begin{tabular}{|c|c|c|c|}
\hline & RPCP - SVP & SVP - IVP & IVP - DVP \\
\hline $7^{\circ}$ nasal & $45 \mu m$ & $54 \mu m$ & $62 \mu m$ \\
\hline $7^{\circ}$ temporal & - & $66 \mu m$ & $54 \mu m$ \\
\hline
\end{tabular}


a. Confocal OIT

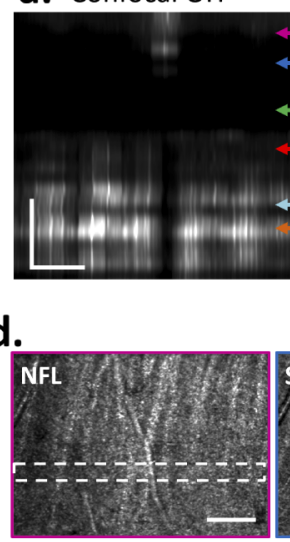

e.

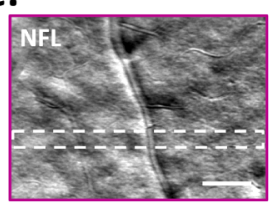

f.

Perfusion Map images
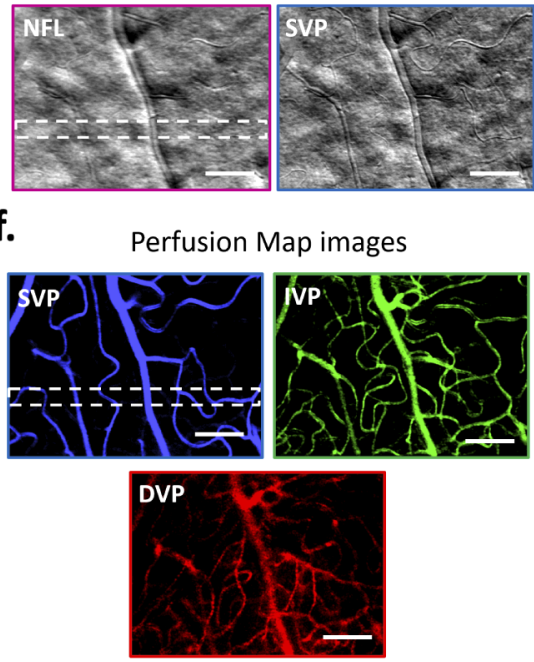

b. Split Detection OIT
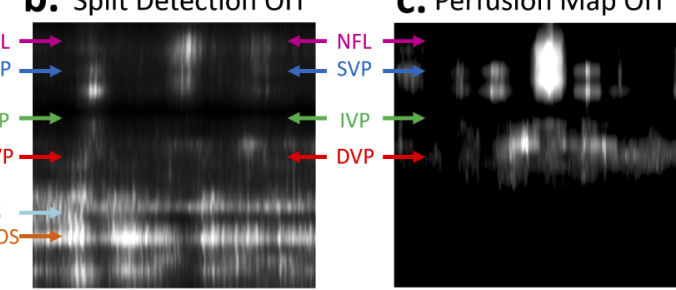

\section{Perfusion Map OIT}

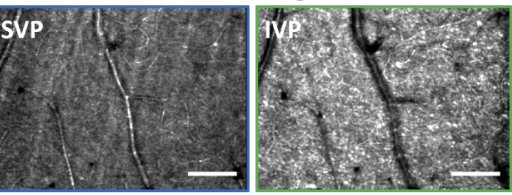

Split Detection images
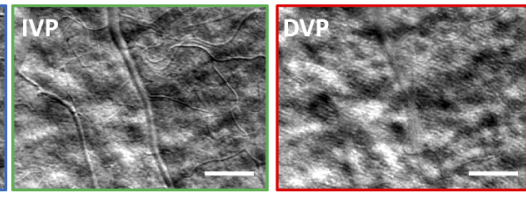

g. $3 D$ Perfusion Map
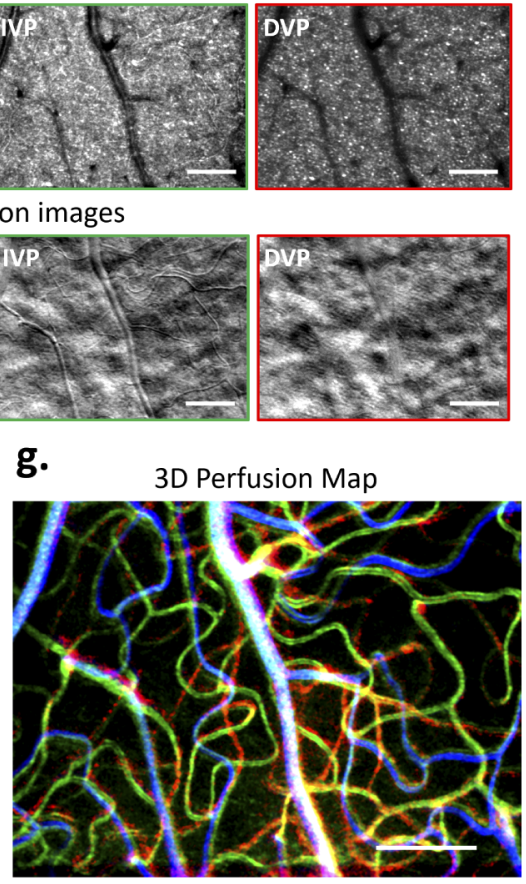

Fig. 5. a-c Tomographic retinal cross-sections generated by, respectively, confocal, split detection, and motion contrast techniques in AO-SLO for the same subject at $7^{\circ}$ Temporal where NFL is less thick and three vascular plexuses can be seen. d-f En-face retinal images obtained when precisely positioning the focal plane at the layers labeled NFL, SVP, IVP and DVP, with the help of the OIT cross-section, using respectively confocal, split-detection and motion contrast techniques. g Composite perfusion map image, revealing the $3 \mathrm{D}$ organization of the retinal vascular network. White-dashed rectangle: Area of the en-face image where OIT cross-sections were extracted. (a) Log scale. (b,c) Linear scale. Scale bar: 100 $\mu \mathrm{m}$. 
mosaic is sharper and presents a higher contrast at the IS focal position (Fig. 6(b,f)) than at the IS/OS focal position (Fig. 6(d,h)). On the other hand, when using the confocal modality, note that the photoreceptor mosaic presents a higher contrast and sharpness at IS/OS focal position (Fig. 6(c,g)) compared to the IS focal position (Fig. 6(a,e)). In Figs. 6(i,j), this same difference of contrast can be noted when looking at the photoreceptor mosaic spatial frequency (pale green column) through the computed PSD of these images.
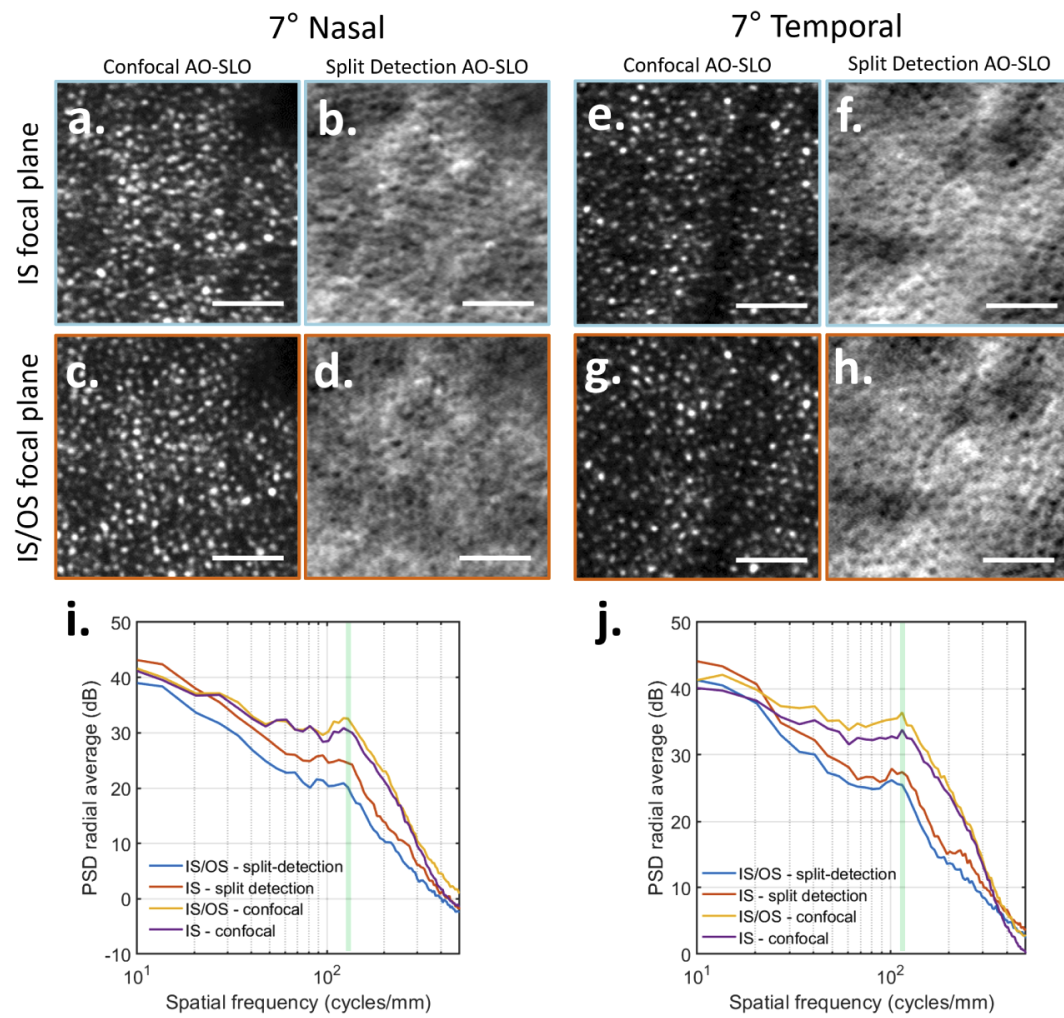

Fig. 6. En-face retinal images obtained after precisely positioning the focal plane at layers IS and IS/OS with the help of OIT cross-section images in Figs. 4,5. (a,e) Confocal AO-SLO at IS focal position. (b,f) Split-detection AO-SLO at IS focal position. (c,g) Confocal AO-SLO at IS/OS focal position. (d,h) Split-detection AO-SLO at IS/OS focal position. (i,j,j) PSD at Nasal and Temporal position respectively. Pale green column outlines the photoreceptor mosaic spatial frequency, where one can notice a gain in contrast when focusing at the IS focus position in the case of split-detection mode, compared to the IS/OS focus position; and the opposite in the case of confocal mode. Scale bar: $50 \mu m$.

Finally, OIT can be used to position the imaging focal plane at the SVP focal position using the original Z-stack, where retinal ganglion cells (RGC) can be visualized in the split detection channel (Fig. 7). Figures 7(a,b) present some examples of RGCs at $7^{\circ}$ Temporal and $7^{\circ}$ Nasal respectively. Since just two offset channels were used to generate the split-detection image, it is not surprising that a contiguous mosaic was not obtained, but only some RGCs were visible [7]. Visible RGCs had a soma diameter varying from $10 \mu \mathrm{m}$ to $20 \mu \mathrm{m}$, which is in the expected range for midget and parasol cells [30]. Figures 7(c,d) compare split-detection images from the same retinal region but at IS and SVP focal positions respectively, where photoreceptor IS mosaic and RGC are visible. Figs. 7(e,f) present their respective PSD. From the PSD, we computed a mean cell density of $15540 \mathrm{cell} / \mathrm{mm}^{2}$ and $9140 \mathrm{cell} / \mathrm{mm}^{2}$ for respectively photoreceptors and RGCs. 
All these findings are consistent with previous in-vivo studies [7,30] and histological data [31]. The SVP being closer to the RGC layer has been previously reported in in-vivo OCT angiography studies $[24,29]$.

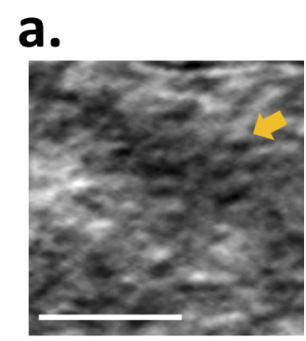

b.

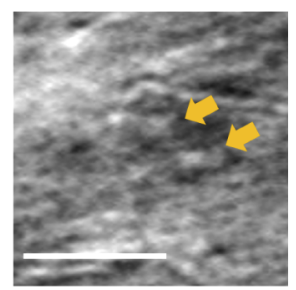

\section{$7^{\circ}$ Temporal}

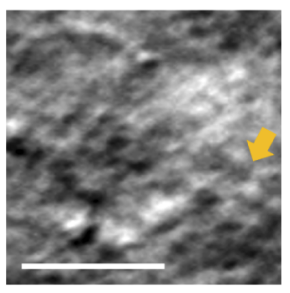

$7^{\circ}$ Nasal
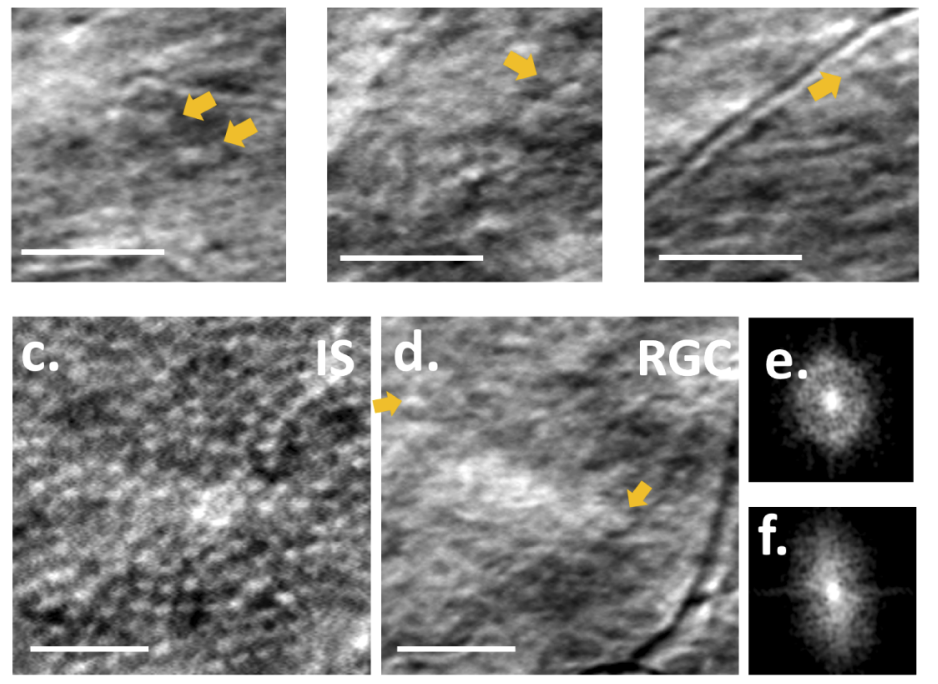

Fig. 7. (a,b) Split-detection retinal images obtained when precisely positioning the focal plane at the SVP layer at $7^{\circ}$ Temporal (a) and $7^{\circ}$ Nasal (b). Yellow arrows outline a few examples of putative midget retinal ganglion cells. The red arrow highlights an example of putative parasol retinal ganglion cell.(c,d) are respectively split-detection images from the same retinal region but at IS and SVP focal positions respectively, where photoreceptor IS mosaic and RGC are visible. (e,f) are the PSD of respectively $(\mathbf{c}, \mathbf{d})$, where the spatial frequency signature of photoreceptor and ganglion cell are visible. Scale bar: $50 \mu m$.

\section{Discussion}

We have introduced Optical Incoherence Tomography (OIT), a completely digital method enabling the generation of in-vivo tomographic retinal cross-sectional images from through focus en-face image stacks. We showed that the OIT procedure can be used on any high numerical aperture adaptive optics incoherent imager, whether scanning or full field, confocal or nonconfocal, making use of back-scattered, multiply-scattered or even fluorescent light, without any hardware modification. While this article discusses the application of OIT to retinal imaging, this method can be extended to other samples (e.g. cornea [32] and skin [33]) and for other high-resolution microscopic/imaging techniques including those used in non-biological samples. Although the axial resolution capacity of the OIT method, limited by the numerical aperture, is far from what 
can be achieved using OCT when applied to the retina, we would like to discuss some interesting assets unique to the OIT method that are complementary to OCT.

\subsection{Focus-guidance for AO-ophthalmoscopes}

Owing to the fact that the axial dimension in OIT cross sections is given as a function of defocus, OIT can be used to precisely position the imaging focal plane in the retina and facilitate non-interferometric imaging of a specific retinal layer. In Fig. 2, we showed that OIT crosssections can help in identifying the focus position to produce images of NFL and IS/OS in both AO-FIO and AO-SLO. In addition, we demonstrated that OIT cross-sections can also be applied to precisely position the focal plane in imaging modalities where users are mostly "blind" focusing because of inherent weak SNR and low contrast, as in the case of autofluorescence (Fig. 2(a)), split-detection (Figs. 4(b), 5(b)), and motion contrast (Figs. 4(c),5(c)) techniques. We demonstrated this particular asset by precisely measuring and positioning the focal position to acquire images of RPE (Fig. 3(b)), perfusion maps from all vascular plexuses (Figs. 4(f),5(f)), and translucent retinal neurons such as photoreceptor IS (Fig. 6) and RGC (Fig. 7), using respectively autofluorescence, motion contrast and split detection techniques.

For optical imaging systems featuring some means to adjust the focus position, the OIT method can be easily implemented to guide focus position during an imaging session as OIT is a completely digital method. One can start by acquiring a fly-through focus movie (also known as Z-stack), generate the patient's OIT cross-section, then use the cross-sectional view and focus information to precisely position the imaging focal plane at the retinal layer of interest. This new procedure avoids loss of time in acquiring images from different depths and selecting the best image sequences afterwards. Although in this paper Z-stacks were generated in a step-wise manner, i.e. by manually changing the focus position and acquiring enough images for each imaging plane in a $600 \mu m \times 600 \mu m$ FOV, Z-stacks could be acquired more rapidly. Firstly, as only a small portion of the FOV from en-face images is necessary to generate the OIT crosssection (about $60 \mu \mathrm{m}$ in one direction), the Z-stack acquisition can be limited to this small area, significantly increasing the acquisition rate. Secondly, instead of using a step-wise acquisition, a fly-through focus (i.e. continuous change of focus position) during image acquisition could be used. Finally, the Z-stack acquisition could be executed in a limited depth range around the retinal layer of interest. For example, in the case of RPE imaging with autofluorescence, where hundreds of en-face images have to be acquired and averaged, the Z-stack could be acquired only around the focal plane where the IS/OS junction looks sharp. Then, the generated OIT cross-section would only include the bright layers corresponding to the IS/OS and the COST (Fig. 2(a)), which are those relevant to RPE layer positioning.

Even though the axial resolution of OIT cross-sections is not as high as OCT, it directly translates the axial resolution of the used imaging system and can be helpful to assess the performance of the AO and the impact of ocular aberrations [4]. Moreover, to precisely measure the focus position, one can use interpolation and fitting methods to achieve a micrometer precision in the retina, similar to what is done in sub-pixel image registration techniques [34].

\subsection{Insensitivity to axial eye motion}

The axial dimension of the OCT is given in terms of optical path difference between the reference and sample arms. This makes OCT highly sensitive to involuntary head and eye motion during image acquisition. Therefore a real-time hardware correction [35] or a post-processing software correction of axial movement are mandatory steps to extract en-face images using both scanning or full-field OCT techniques [36,37]. Residual axial motion, after correction, can even degrade the axial resolution of OCT cross-sections [36].

On the other hand, OIT is insensitive to axial motion. Nevertheless, it is highly sensitive to the temporal evolution of the defocus, mainly introduced by the lens accommodation 
dynamics. Defocus dynamics prevent generation of OIT cross-sections. The temporal evolution of accommodation and defocus in the eye [3] is less important than the eye and head axial movement during fixation [35], making it easier to control the former. Here, to tackle defocus dynamics, we used high AO-loop rates to correct for dynamic aberrations [4], and we performed accommodation paralysis by introducing drops. Both solutions are commonly found in imaging protocols for high-resolution retinal imaging.

\subsection{Guiding retinal laser surgery}

The lack of sensitivity to axial motion together with controlled accommodation dynamics makes OIT a highly valuable tool to accurately focus light in a sample. One promising application would be using OIT to guide focus position in laser photocoagulation retinal surgery [38-40]. Indeed, using both $\mathrm{AO}$ with a high numerical aperture to achieve a suitable 3D laser confinement in the retina and OIT to precisely position the laser impact at the diseased tissue, would represent a considerable advance towards a safe and controlled laser surgery of the retina, avoiding damage to neighboring healthy tissues $[41,42]$.

\subsection{Using multiply scattered light to generate retinal cross-sections}

Multiply scattered light (or nonconfocal) imaging modalities such as dark-field, offset aperture and split detection, widely applied in AO-SLO [5], and recently introduced for AO-FIO [10,43], provide excellent contrast for blood vessels, mural cells $[8,10]$ and translucent retinal structures [6,7], which are poorly or not visualized in back-scattered light imaging systems. Unlike OCT, in which the coherent detection limits the use of multiply scattered light, we showed that OIT can make use of incoherent light, generating, for the first time to our knowledge, a retinal cross-sectional view using multiply-scattered light, here in split-detection mode (Figs. 4(b),5(b)). The use of multiply-scattered light can also be extended to other techniques, such as offset aperture, multi-offset [7] and dark-field techniques. Moreover, fluorescent light could also be used to generate a cross-section, which does not produce any signal in OCT. Comparing different modalities, along with their en-face images, may help to elucidate the cellular origin of the observed features on en-face images [44] and/or OIT images.

One example is the origin of the observed photoreceptor mosaic when using split-detection technique, which is commonly attributed to the photoreceptor inner segment (IS) [6]. Figs. 4(b), 5(b) shows a retinal layer, just above IS/OS, which gets brighter in split-dectection mode compared to confocal. We denominated this layer IS. By positioning the focal plane at this layer, we were able to acquire a sharper photoreceptor mosaic than at the IS/OS focal position. Although not conclusive, our findings support the hypothesis that the cellular origin of the split detection photoreceptor mosaic image may come from the IS [6]. Further studies are still necessary and OIT could be a highly valuable tool to help elucidate this question.

Another example of how a multimodal approach can help in interpreting OIT cross-sections can be seen in Fig. 4. While the split-detection OIT cross-section revealed bright layers corresponding to different vascular plexus, these same layers can also be seen in confocal OIT cross-sections, but more dimly and with lower contrast. En-face images also show the same trend, as capillaries are more highly contrasted in the split-detection modality than in confocal. Note that similar layer arrangement and intensity can be seen in Fig. 2(a), which may explain the origin of intermediate bands (1) and (2), i.e. corresponding to the IVP and DVP.

Depending on the properties of the tissue and the local anatomy, the retina scatters light in different directions and angles [45]. OIT could help to determine the scattering direction of different retinal features for various focus positions when applied to multi-offset techniques $[7,45]$. Moreover, OIT could help to further enhance the contrast of the retinal ganglion cell layer by finding the optimal focal position in multi-offset imaging [7]. 
OIT makes use of its high lateral resolution to determine the depth location of small features in the retina. One example was given in Visualization 2, where interconnecting capillaries linking different vascular plexus could be identified and their depth location determined when using an AO-FIO. Unlike confocal OIT (Figs. 2(a),4(a)), signal from interconnecting capillaries can also be identified in split-detection OIT (Fig. 4(b)). The fact that interconnecting capillaries can only be seen in OIT generated from AO-FIO and split-detection AO-SLO images may infer that their contrast is highly enhanced by multiply scattered light, which is mostly filtered out in confocal AO-SLO. This hypothesis is reinforced by the work done in [45] with a rapidly configurable aperture enabling multi-offset AO-SLO imaging. Although not commented by the authors, their Figure 5 shows interconnecting capillaries appearing and disappearing depending on the orientation of the collected scattered light, which is not visible in the confocal modality.

\subsection{Limitations}

When applied to in-vivo retinal imaging, the axial sectioning of OIT may vary depending on different aspects. First, it presents an inter-subject variation, as pupil diameter (even with induced pupil dilation), eye length and dynamic aberrations may vary from one subject to another. Both pupil diameter and dynamic aberrations may be critical in the case of diseased patients. High-order dynamic aberrations can provoke a significant loss of axial resolution. This issue can be considerably minimized by adopting a high AO-loop rate (higher than $50 \mathrm{~Hz}$ ) [4]. Secondly, unlike OCT, accommodation paralysis has to be applied to increase the axial sectioning capacity of OIT method, common to all AO techniques. Thirdly, the axial sectioning capacity may vary according to the selected cut-off frequency, which has to take into account the OIT contrast (Fig. 1(e)) and the spatial frequency content of the retinal features of interest. Lastly, when using a step-wise manner to acquire the Z-stack, the defocus step has to be carefully chosen and computed to appropriately sample the axial direction without losing resolution. Throughout this paper, defocus steps of $30 \mu m$ (Fig. 2(c)), $20 \mu m$ (Fig. 2(a)) and $15 \mu m$ (Figs. 4,5) were used, the latter enabling identification of more layers than the former ones.

To get absolute and precise values of axial depth position, a first calibration step may be necessary to take into account the variation of the optical axial magnification in a non-telecentric system and the eye length of the examined subject. However, focus guidance for precise imaging, or focus in a sample, can be done with a relative measurement, as it was done in this paper, if the same system is used to generate the OIT cross-section then acquire the en-face retinal images. Finally, another drawback can be pupil misalignment during Z-stack acquisition, which leads to loss of photons.

\section{Conclusion}

We presented Optical Incoherence Tomography (OIT): a completely digital method to generate tomographic retinal cross-sections from en-face through-focus image stacks acquired with any high numerical aperture non-interferometric AO-ophthalmoscope. We demonstrated that OIT can be applied to retinal imaging modalities such as AO-FIO, AO-SLO, split-detection, offset aperture, and motion contrast without any hardware modification. We showed that even though OIT presents a poorer axial resolution compared to OCT, most of the retinal layers commonly found in OCT can also be identified in OIT cross-sectional images. While OCT is sensitive to the optical path difference between the reference and the sample arms, we showed that OIT is sensitive to focus position, making it a highly valuable tool to guide focus position. We explore this property to guide focus position in cases where the user is "blind" focusing, allowing precise imaging of retinal pigment epithelium, the vascular plexuses and translucent retinal neurons, such as photoreceptor inner segment and retinal ganglion cells, using respectively autofluorescence, motion contrast and split detection techniques. All the presented assets specific to OIT may play an important role in in-vivo retinal imaging, paving the way towards more rapid imaging sessions, 
a more precise focus positioning, and a better understanding of some particular retinal features, especially when using multiply-scattered light.

\section{Funding}

Conseil Régional, Île-de-France fund SESAME 4D-EYE (EX047007); Agence Nationale de la Recherche (CLOVIS3D grant, ANR-14-CE17-0011, IHU FOReSIGHT, ANR-18-IAHU-0001); European Research Council Hemholtz grant (610110).

\section{Acknowledgments}

The authors want to thank Laurent Mugnier, Cyril Petit, Frédéric Cassaing, Yann Lai-Tim and Antoine Chen for fruitful discussions on the OIT method, and Ethan Rossi for insights and discussion about retinal ganglion cells.

\section{Disclosures}

P.M. and S.M. are listed as inventors on a patent application (FR 1904271) related to the work presented in this manuscript. All other authors have nothing to disclose.

\section{References}

1. E. Gofas-Salas, P. Mecê, C. Petit, J. Jarosz, L. M. Mugnier, A. M. Bonnefois, K. Grieve, J. Sahel, M. Paques, and S. Meimon, "High loop rate adaptive optics flood illumination ophthalmoscope with structured illumination capability," Appl. Opt. 57(20), 5635-5642 (2018).

2. A. Roorda and J. L. Duncan, "Adaptive optics ophthalmoscopy,” Annu. Rev. Vis. Sci. 1(1), 19-50 (2015).

3. J. Jarosz, P. Mecê, J.-M. Conan, C. Petit, M. Paques, and S. Meimon, "High temporal resolution aberrometry in a 50-eye population and implications for adaptive optics error budget," Biomed. Opt. Express 8(4), 2088-2105 (2017).

4. P. Mecê, E. Gofas-Salas, C. Petit, F. Cassaing, J. Sahel, M. Paques, K. Grieve, and S. Meimon, "Higher adaptive optics loop rate enhances axial resolution in nonconfocal ophthalmoscopes," Opt. Lett. 44(9), 2208-2211 (2019).

5. S. A. Burns, A. E. Elsner, K. A. Sapoznik, R. L. Warner, and T. J. Gast, "Adaptive optics imaging of the human retina," Prog. Retinal Eye Res. 68, 1-30 (2019).

6. D. Scoles, Y. N. Sulai, C. S. Langlo, G. A. Fishman, C. A. Curcio, J. Carroll, and A. Dubra, "In vivo imaging of human cone photoreceptor inner segments," Invest. Ophthalmol. Visual Sci. 55(7), 4244-4251 (2014).

7. E. A. Rossi, C. E. Granger, R. Sharma, Q. Yang, K. Saito, C. Schwarz, S. Walters, K. Nozato, J. Zhang, T. Kawakami, W. Fischer, L. R. Latchney, J. J. Hunter, M. M. Chung, and D. R. Williams, "Imaging individual neurons in the retinal ganglion cell layer of the living eye," Proc. Natl. Acad. Sci. 114(3), 586-591 (2017).

8. T. Y. Chui, D. A. Van Nasdale, and S. A. Burns, "The use of forward scatter to improve retinal vascular imaging with an adaptive optics scanning laser ophthalmoscope," Biomed. Opt. Express 3(10), 2537-2549 (2012).

9. K. Grieve, E. Gofas-Salas, R. D. Ferguson, J. A. Sahel, M. Paques, and E. A. Rossi, "In vivo near-infrared autofluorescence imaging of retinal pigment epithelial cells with $757 \mathrm{~nm}$ excitation," Biomed. Opt. Express 9(12), 5946-5961 (2018).

10. E. Gofas-Salas, P. Mecê, L. Mugnier, A. M. Bonnefois, C. Petit, K. Grieve, J. Sahel, M. Paques, and S. Meimon, "Near infrared adaptive optics flood illumination retinal angiography," Biomed. Opt. Express 10(6), 2730-2743 (2019).

11. M. Miura and A. E. Elsner, "Three dimensional imaging in age-related macular degeneration," Opt. Express 9(9), 436-443 (2001).

12. A. Elsner, M. Miura, S. Burns, E. Beausencourt, C. Kunze, L. Kelley, J. Walker, G. Wing, P. Raskauskas, D. Fletcher, Q. Zhou, and A. Dreher, "Multiply scattered light tomography and confocal imaging: detecting neovascularization in age-related macular degeneration," Opt. Express 7(2), 95-106 (2000).

13. Y. Zhang, L. Liu, W. Gong, H. Yu, W. Wang, C. Zhao, P. Wang, and T. Ueda, "Autofocus system and evaluation methodologies: a literature review," Sens. Mater. 30, 1165-1174 (2018).

14. D. Alonso-Caneiro, D. M. Sampson, A. L. Chew, M. J. Collins, and F. K. Chen, "Use of focus measure operators for characterization of flood illumination adaptive optics ophthalmoscopy image quality," Biomed. Opt. Express 9(2), 679-693 (2018).

15. A. Lazareva, P. Liatsis, and F. G. Rauscher, "Hessian-log filtering for enhancement and detection of photoreceptor cells in adaptive optics retinal images," J. Opt. Soc. Am. A 33(1), 84-94 (2016).

16. D. Huang, E. A. Swanson, C. P. Lin, J. S. Schuman, W. G. Stinson, W. Chang, M. R. Hee, T. Flotte, K. Gregory, C. A. Puliafito, and J. G. Fujimoto, "Optical coherence tomography," Science 254(5035), 1178-1181 (1991).

17. D. Lim, K. K. Chu, and J. Mertz, "Wide-field fluorescence sectioning with hybrid speckle and uniform-illumination microscopy," Opt. Lett. 33(16), 1819-1821 (2008). 
18. P. Mecê, “Optical Incoherence Tomography (OIT) software,” (2020). https://doi.org/10.5281/zenodo.3888203.

19. A. Roorda, F. Romero-Borja, W. J. Donnelly III, H. Queener, T. J. Hebert, and M. C. Campbell, "Adaptive optics scanning laser ophthalmoscopy," Opt. Express 10(9), 405-412 (2002).

20. M. Born and E. Wolf, Principles of Optics: Electromagnetic Theory of Propagation, Interference and Diffraction of Light (Elsevier, 2013).

21. P. Mecê, E. Gofas Salas, C. Petit, K. Grieve, C. Chabrier, M. Paques, and S. Meimon, "Visualizing and enhancing axial resolution in nonconfocal adaptive optics ophthalmoscopy," Proc. SPIE 10858, 108580P (2019).

22. P. Mecê, J. Jarosz, J.-M. Conan, C. Petit, K. Grieve, M. Paques, and S. Meimon, "Fixational eye movement: a negligible source of dynamic aberration," Biomed. Opt. Express 9(2), 717-727 (2018).

23. M. Mujat, A. Patel, N. Iftimia, J. D. Akula, A. B. Fulton, and R. D. Ferguson, "High-resolution retinal imaging: enhancement techniques," in Ophthalmic Technologies XXV, vol. 9307 (International Society for Optics and Photonics, 2015), p. 930703.

24. J. Campbell, M. Zhang, T. Hwang, S. Bailey, D. Wilson, Y. Jia, and D. Huang, "Detailed vascular anatomy of the human retina by projection-resolved optical coherence tomography angiography," Sci. Rep. 7(1), 42201 (2017).

25. S. Fouquet, O. Vacca, F. Sennlaub, and M. Paques, "The 3D retinal capillary circulation in pigs reveals a predominant serial organization,” Invest. Ophthalmol. Visual Sci. 58(13), 5754-5763 (2017).

26. R. F. Cooper, M. A. Wilk, S. Tarima, and J. Carroll, "Evaluating descriptive metrics of the human cone mosaic," Invest. Ophthalmol. Visual Sci. 57(7), 2992-3001 (2016).

27. Z. Liu, O. P. Kocaoglu, and D. T. Miller, "3D imaging of retinal pigment epithelial cells in the living human retina," Invest. Ophthalmol. Visual Sci. 57(9), OCT533 (2016).

28. C. Lavia, P. Mecê, M. Nassisi, S. Bonnin, J. Marie-Louise, A. Couturier, A. Erginay, R. Tadayoni, and A. Gaudric, "Retinal capillary plexus pattern and density from fovea to periphery measured in healthy eyes with swept-source optical coherence tomography angiography," Sci. Rep. 10(1), 1474 (2020).

29. T. Hirano, K. Chanwimol, J. Weichsel, T. Tepelus, and S. Sadda, "Distinct retinal capillary plexuses in normal eyes as observed in optical coherence tomography angiography axial profile analysis," Sci. Rep. 8(1), 9380 (2018).

30. Z. Liu, K. Kurokawa, F. Zhang, J. J. Lee, and D. T. Miller, "Imaging and quantifying ganglion cells and other transparent neurons in the living human retina," Proc. Natl. Acad. Sci. 114(48), 12803-12808 (2017).

31. C. A. Curcio and K. A. Allen, "Topography of ganglion cells in human retina," J. Comp. Neurol. 300(1), 5-25 (1990).

32. I. Jalbert, F. Stapleton, E. Papas, D. Sweeney, and M. Coroneo, "In vivo confocal microscopy of the human cornea," Br. J. Ophthalmol. 87(2), 225-236 (2003).

33. M. Rajadhyaksha, A. Marghoob, A. Rossi, A. C. Halpern, and K. S. Nehal, "Reflectance confocal microscopy of skin in vivo: From bench to bedside," Lasers Surg. Med. 49(1), 7-19 (2017).

34. D. Gratadour, L. Mugnier, and D. Rouan, "Sub-pixel image registration with a maximum likelihood estimatorapplication to the first adaptive optics observations of arp 220 in the 1 band," Astron. Astrophys. 443(1), 357-365 (2005).

35. P. Mecê, J. Scholler, K. Groux, and C. Boccara, "High-resolution in-vivo human retinal imaging using full-field oct with optical stabilization of axial motion," Biomed. Opt. Express 11(1), 492-504 (2020).

36. R. S. Jonnal, O. P. Kocaoglu, R. J. Zawadzki, S.-H. Lee, J. S. Werner, and D. T. Miller, "The cellular origins of the outer retinal bands in optical coherence tomography images," Invest. Ophthalmol. Visual Sci. 55(12), 7904-7918 (2014).

37. P. Mecê, K. Groux, J. Scholler, O. Thouvenin, M. Fink, K. Grieve, and C. Boccara, "Curved-full-field oct for high-resolution imaging of living human retina over a large field-of-view," arXiv preprint arXiv:2001.06893 (2020)

38. M. Kernt, R. E. Cheuteu, S. Cserhati, F. Seidensticker, R. G. Liegl, J. Lang, C. Haritoglou, A. Kampik, M. W. Ulbig, and A. S. Neubauer, "Pain and accuracy of focal laser treatment for diabetic macular edema using a retinal navigated laser (navilas)," Clin. Ophthalmol. 6, 289 (2012).

39. B. Liesfeld, K.-U. Amthor, D. Dowell, U. Weber, and W. Teiwes, "Navigating comfortably across the retina," in World Congress on Medical Physics and Biomedical Engineering, September 7-12, 2009, Munich, Germany (Springer, 2009), pp. 243-246.

40. M. Paques, E. Philippakis, C. Bonnet, S. Falah, S. Ayello-Scheer, S. Zwillinger, J.-F. Girmens, and B. Dupas, "Indocyanine-green-guided targeted laser photocoagulation of capillary macroaneurysms in macular oedema: a pilot study,” Br. J. Ophthalmol. 101(2), 170-174 (2017).

41. P. Mecê, C. Petit, E. G. Salas, L. Mugnier, K. Grieve, C. Chabrier, J. A. Sahel, M. Paques, and S. Meimon, "What can adaptive optics do for laser photocoagulation?” Invest. Ophthalmol. Visual Sci. 59, 6194 (2018).

42. P. Mecê, "4D exploration of the retina for adaptive optics-assisted laser photocoagulation," (2018). https://www.researchgate.net/publication/342123596.

43. S. Meimon, E. G. Salas, P. Mecě, K. Grieve, J. A. Sahel, and M. Paques, "Manipulation of the illumination geometry on adaptive optics (AO) flood illumination ophthalmoscope (FIO) for dark field imaging of the retina," Invest. Ophthalmol. Visual Sci. 59, 4641 (2018).

44. A. Guevara-Torres, D. Williams, and J. Schallek, "Origin of cell contrast in offset aperture adaptive optics ophthalmoscopy," Opt. Lett. 45(4), 840-843 (2020).

45. K. A. Sapoznik, T. Luo, A. De Castro, L. Sawides, R. L. Warner, and S. A. Burns, "Enhanced retinal vasculature imaging with a rapidly configurable aperture," Biomed. Opt. Express 9(3), 1323-1333 (2018). 